\title{
Discovery of $5.16 \mathrm{~s}$ pulsations from the isolated neutron star RBS 1223
}

\author{
V. Hambaryan ${ }^{1}$, G. Hasinger ${ }^{1}$, A. D. Schwope ${ }^{1}$, and N. S. Schulz ${ }^{2}$ \\ 1 Astrophysikalisches Institut Potsdam, An der Sternwarte 16, 14482 Potsdam, Germany \\ 2 Massachusetts Institute of Technology, Center for Space Research, 70 Vassar Street, Cambridge, \\ MA 02139, USA
}

Received 11 May 2001 / Accepted 4 October 2001

\begin{abstract}
The isolated neutron star candidate RBS 1223 was observed with the Advanced CCD Imaging Spectrometer aboard the Chandra X-ray observatory on 2000 June 24. A timing analysis of the data yielded a periodic modulation with a period $P=5.1571696_{-1.36 \times 10^{-4}}^{+1.57 \times 10^{-4}}$ s. Using ROSAT HRI archived observations we detected a period $P=5.1561274 \pm 4.4 \times 10^{-4} \mathrm{~s}$ and determined period derivative $\dot{P}=(0.7-2.0) \times 10^{-11} \mathrm{~s} \mathrm{~s}^{-1}$. The detection of this period and $\dot{P}$ indicates that RBS 1223 has a "characteristic" age of $6000-12000$ years and huge magnetic field at the surface $\left(B_{\text {dipole }} \approx(1.7-3.2) \times 10^{+14} \mathrm{G}\right)$ typical for anomalous X-ray pulsars (AXPs).
\end{abstract}

Key words. stars: neutron - stars: individual: RBS1223 - X-rays: stars

\section{Introduction}

Neutron stars (NS) supposedly represent a non-negligible fraction of $\sim 1 \%$ of all stars in the Galaxy, with a total number as high as $10^{8}-10^{9}$ (e.g. Narayan \& Ostriker 1990). Isolated NS represent the bulk of the population and soon after their birth may appear as radio pulsars. This pulsar phase could last some $10^{7} \mathrm{yr}$ and these NS are observable, if the beaming conditions are favorable. At formation NS are very hot $\left(T \sim 10^{11} \mathrm{~K}\right)$, and they may give off thermal radiation while cooling.

Depending on some model assumptions, a few hundred to several thousand accreting isolated NSs were expected to be detectable in the ROSAT All-Sky Survey (Treves \& Colpi 1991; Blaes \& Madau 1993; Neuhäuser \& Trümper 1999). However, to date only $6-7$ good candidates (Treves et al. 2000; Motch 2000) for isolated NSs were discovered in ROSAT data (RXJ1856.6-3754, Walter 1996; RX J0720.4-3125, Haberl et al. 1997; RX J0806.4-4123, Haberl et al. 1998; 1RXS J130848.6+212708 $\equiv$ RBS 1223, Schwope et al. 1999; RX J1605.3+3249, Motch \& Haberl 1999; and RX J0420.0-5022, Haberl et al. 1999). They can be exclusively described by their similar X-ray properties, i.e. they have soft X-ray spectra which are well represented by pure blackbody emission with temperatures $k T$ between 50 and $120 \mathrm{eV}$, and extremely high X-ray to optical flux ratios, $\log \left(f_{x} / f_{\text {opt }}\right)$ of $4-5.5$ (see tables in Treves et al. 2000; Motch 2000).

Send offprint requests to: V. Hambaryan,

e-mail: vhambaryan@aip.de
Most probably, these best candidates constitute the bright end of the $\log N-\log S$ distribution of X-ray detected isolated NSs (Neuhäuser \& Trümper 1999), suggesting that the smaller number of observed accreting old neutron stars than those theoretically expected is mainly caused by their larger space velocities compared to the previously assumed velocity distribution. This conclusion is supported by the recent discovery of a proper motion for RX J1856.6-3754 (Walter 2001). Two of these objects (RX J0720.4-3125, RX J0420.0-5022) exhibit pulsations with remarkably long periods of $8.39 \mathrm{~s}$ and $22.7 \mathrm{~s}$.

The point source RBS 1223 (1RXS J130848.6+212708) was detected in the ROSAT All-Sky Survey (1RXS catalogue, Voges et al. 1999) and studied as a candidate isolated neutron star (Schwope et al. 1999) during the program of optical identifications of bright $\left(>0.2\right.$ PSPC cts s$\left.^{-1}\right)$, high-galactic-latitude $\left(|b|>30^{\circ}\right)$ X-ray sources (ROSAT Bright Survey - RBS, Schwope et al. 2000).

A pointed ROSAT HRI observation (Schwope et al. 1999) determined the position of the source to be $\alpha(\mathrm{J} 2000)=13^{\mathrm{h}} 08^{\mathrm{m}} 48.17^{\mathrm{s}}, \quad \delta(\mathrm{J} 2000)=$ $21^{\circ} 27^{\prime} 07.5^{\prime \prime}\left( \pm 1.6^{\prime \prime}\right)$. The corresponding Galactic coordinates are $l=339^{\circ}, b=+83^{\circ}$. Deep optical observations with Keck-II failed to detect an optical counterpart down to a limiting magnitude of $m_{B} \sim 26$, thereby placing a lower limit of $\sim 10000$ on the X-ray to optical flux ratio (Schwope et al. 1999).

In this paper we report the detection of pulsations from the isolated neutron star RBS 1223 based on recent 
observations with the Chandra X-ray observatory and ROSAT archival observations.

\section{Observations and data reduction}

The point source RBS 1223 was observed with the Advanced CCD Imaging Spectrometer (back illuminated S3 chip) on board the Chandra X-ray observatory on 2000 June 24 , for a total of $\sim 10 \mathrm{ksec}$. The source was placed at the farthest corner of the back-illuminated CCD $\mathrm{S}$ array, close to the read out of node 3 in order to mitigate pile-up and charge transfer inefficiency effects. Only a small part $(1 / 8)$ of the CCD was readout to additionally avoid pile-up and increase the time resolution to $\sim 0.5 \mathrm{~s}$.

We have used level 2 ACIS event data, retaining only "standard" grades 0, 2, 3, 4 and 6 thus discarding events with very large pulse heights as due to cosmic rays.

An inspection of an X-ray image in chip coordinates (rather than sky coordinates in which the dithering of space-craft has been accounted for) showed no "bad" pixels or columns in the source data. Examination of the time dependence of the background did not reveal periods of high background during the observation.

To identify point sources in the field (Fig. 1), we used the routine CELLDETECT provided by the Chandra Interactive Analysis of Observations (CIAO 2.0) software package. We determined the position of RBS 1223 to be $\alpha(\mathrm{J} 2000)=13^{\mathrm{h}} 08^{\mathrm{m}} 48.26^{\mathrm{s}}, \delta(\mathrm{J} 2000)=21^{\mathrm{o}} 27^{\prime} 06.75^{\prime \prime}$, compatible with the position found in previous ROSAT HRI observations (see Sect. 1).

\subsection{Timing analysis}

\subsubsection{Chandra X-ray observatory ACIS-S}

First of all, we performed a timing analysis of Chandra X-ray observatory data in order to search for periodic modulations. We extracted $\sim 7840$ ACIS-S source photon events (Fig. 1), which we corrected for barycenter using the "axBary" programme, available at ftp: //asc.harvard.edu.

An application of various methods (e.g. Scargle 1982; Buccheri et al. 1983) for periodicity search indicates a highly significant detection of a periodic signal at $5.157 \mathrm{~s}$.

To evaluate the pulsation frequency more precisely, and find its uncertainty, we employed the Gregory-Loredo (GL) Bayesian method (Gregory \& Loredo 1992, 1993, 1996). This method is designed for the detection of a periodic signal of unknown shape and period, assuming a Poisson sampling distribution. To compare different models (how the data favor a periodic model of a given frequency $f$ with $m$ phase bins over the constant rate model) it calculates a frequency-dependent Bayesian odds ratios $\left(O_{m}(f)\right)$ (for details, see Gregory \& Loredo 1992).

We implemented the GL method, taking the maximum number of phase bins equal to 15 and surveyed the frequency range $0.01-0.25 \mathrm{~Hz}$. The odds ratio as a function of frequency is shown in Fig. 2 (left panel). As a most

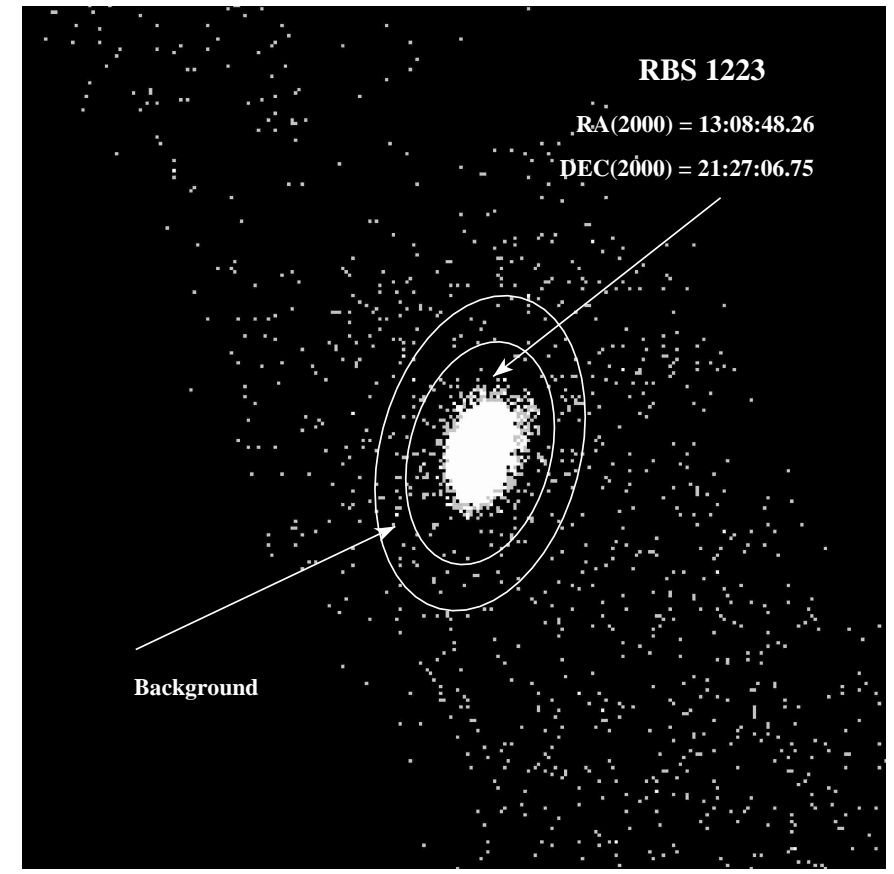

Fig. 1. Chandra ACIS-S image of RBS 1223. Inner ellipse corresponds to the $5 \sigma$ radius of the source (available from source detection procedure, CIAO routine CELLDETECT) and elliptical annuli to the background covering an equal area.

probable value for the period we took the mode of the posterior probability distribution function (PDF).

The maximum value, $O_{\mathrm{m}}^{\max }=6.16 \times 10^{10}$, is at $f_{0}=$ $0.19390681 \mathrm{~Hz}$. We computed the uncertainty of $f_{0}$ at $68 \%$ confidence level ("posterior bubble") using the full Bayesian posterior PDF. It is equal to $\delta f={ }_{-7.913}^{+3.103} \mu \mathrm{Hz}$. Thus, we finally derive the period of the detected pulsations and the uncertainties to be

$P_{\text {Chandra }}=5.1571696_{-1.36 \times 10^{-4}}^{+1.57 \times 10^{-4}} \mathrm{~s}$

at the epoch of 51719.949676196 MJD.

The light curve extracted at $f=f_{0}$ (Fig. 3 left panel) reveals one broad pulse per period. The semi-amplitude modulation,

$A_{\text {Chandra }} \equiv \frac{C R_{\max }-C R_{\min }}{C R_{\max }+C R_{\min }}$,

where $C R$ is the count rate, is equal to $23 \% \pm 5 \%$. Assuming that the detected signal is sinusoidal we estimate that the pulsed fraction intrinsic to the source is $20 \% \pm 2 \%$ (Brazier 1994).

\subsubsection{ROSAT HRI}

The field of RBS 1223 has also been observed with the ROSAT HRI on June 13, 1997 (ROR 703348) and on January 10, 1998 (ROR 704082) for a net exposure time of $2218 \mathrm{~s}$ and $4938 \mathrm{~s}$, correspondingly.

We also performed a timing analysis of the ROSAT HRI observations. In both cases we used a $12^{\prime \prime}$ radius 

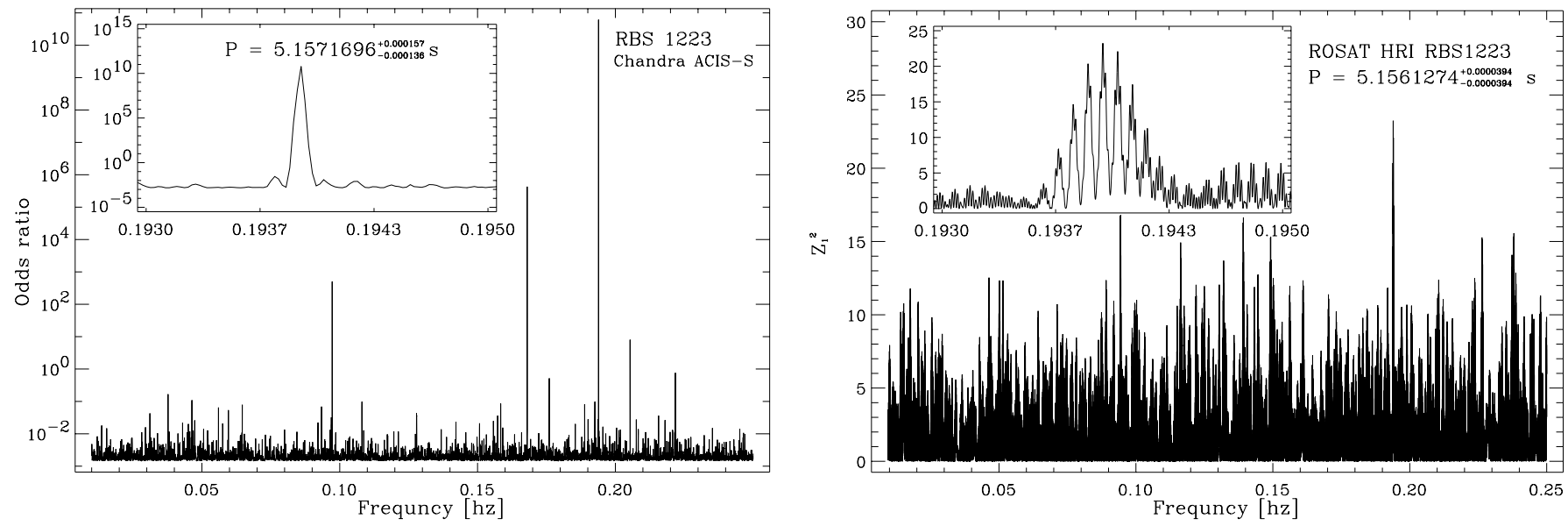

Fig. 2. Periodogrammes of RBS 1223: Chandra ACIS-S (left) and ROSAT HRI (right) observations.

circular region to extract the source photons. This radius encompassed $85 \%$ of the events in the ROSAT point spread function. The extracted data sets consist of 266 and 521 counts, respectively. Photon arrival times were solar barycenter corrected, as implemented in the Extended Scientific Analysis Software System (Zimmerman et al. 1998).

We analyzed the $\sim 266 \mathrm{HRI}$ (ROR 703348) photons from RBS 1223 for periodicities in the X-ray flux, but did not detect any significant signal using a Rayleigh $Z_{1}^{2}$ test (Buccheri et al. 1983). However, a similar analysis of the second data set (ROR 704082, consisting of 521 photons) revealed a periodic modulation at $0.19394401 \mathrm{~Hz}$ (Fig. 2 right panel).

As Fig. 2 shows, the significance of detection is not very high $\left(Z_{1}^{2}=23.2\right)$ owing to the small number of registered photons. Moreover, the power spectrum is quite complicated; it consists of a central peak accompanied by many side lobes owing to the data gaps. This makes the computation of a credible region difficult, since the posterior PDF obtained by an application of the GL method is not unimodal, unlike in the case of the Chandra observations. We determined the uncertainty of this period measurement of RBS 1223 through simulations, based on the measured pulsed fraction $\sim 0.3$ and count rate $\sim 0.1 \mathrm{~s}^{-1}$. Each of our 10000 simulated data sets (photon arrival times) was generated from a sinusoidal light curve and obeyed Poissonian statistics. Each simulated data set had the same characteristics as the observed data in terms of number of registered counts, spanned time, good time intervals, data gaps, and was analyzed exactly in the same way. The dispersion in the obtained period distribution was adopted as an estimate of the uncertainty in our measurement of the period of RBS 1223: $\delta P= \pm 3.949 \times 10^{-5} \mathrm{~s}$.

An error estimate can be derived alternatively from a more general approach In general, the accuracy of a frequency determination in a periodogram depends on the total observation time $T_{\text {total }}$, the number of measurements $N$ and the signal-to-noise ratio (see e.g. Jaynes 1987; Bretthorst 1988; Kovacs 1981).
According to these authors, the theoretical accuracy for determination of the frequency of a single steady sinusoid $\left(d_{i}=A \cos \left(2 \pi f t_{i}\right)+e\left(t_{i}\right)\right.$, single sine wave plus noise $e\left(t_{i}\right)$ of standard deviation $\left.\sigma\right)$ is

$\delta f=\frac{1.1 \sigma}{A \cdot T_{\text {total }} \cdot \sqrt{N}} \mathrm{~Hz}$.

Hence, for the ROSAT observation of RBS 1223 (ROR 704082) we get: $T_{\text {total }} \approx 70 \mathrm{ksec}, N=20$ (number of bins in the phase folded light curve) and signal-to-noise ratio $A / \sqrt{2} \sigma \sim 3$ (see Fig. 3, right panel). This leads to an optimistic estimate of accuracy of the period of:

$\delta P_{\text {optimistic }}= \pm 4.4 \times 10^{-5} \mathrm{~s}$,

while a pessimistic estimate is:

$\delta f=\frac{1}{2 T_{\text {total }}} \mathrm{Hz}$ (Rayleigh resolving power)

$\delta P_{\text {pessimistic }}= \pm 3.8 \times 10^{-4} \mathrm{~s}$.

Thus, we finally derive the period of the detected pulsations in the ROSAT HRI observations

$P_{\text {ROSAT }}=5.1561274 \pm 3.8 \times 10^{-4} \mathrm{~s}$

at the epoch of 50824.311057318 MJD.

This determination allows to calculate a period derivative and its uncertainty

$\dot{P}=1.35_{-0.67}^{+0.69} \times 10^{-11} \mathrm{~s} \mathrm{~s}^{-1}$,

if we adopt the pessimistic error estimate of the accuracy for period determination of RBS 1223 of ROSAT HRI observations $\left(\delta P_{\text {pessimistic }}\right)$.

\subsection{Spectral analysis}

Since the Chandra X-ray observatory ACIS-S detectors have intrinsic energy resolution and the source spectrum has sufficient number counts (count rate $\sim 0.82 \pm$ $0.02 \mathrm{cts}^{-1}$ ), we were able to examine the spectral 

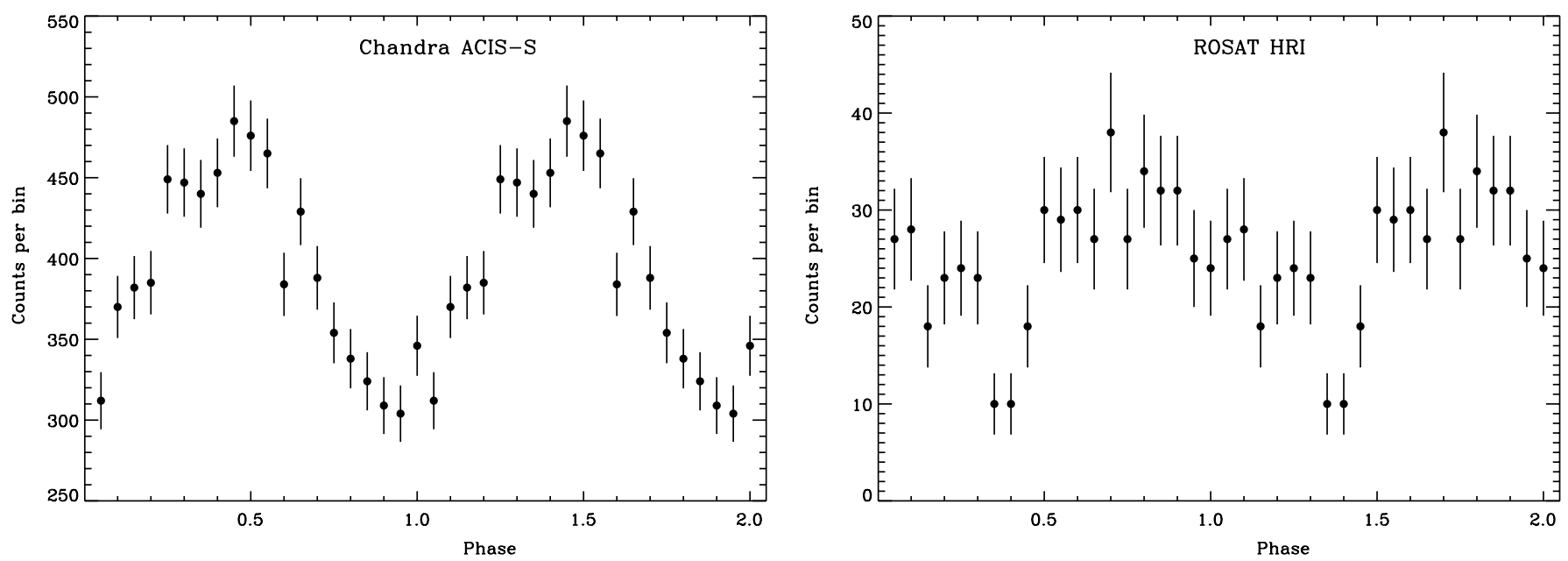

Fig. 3. Phase folded light curves of RBS 1223: Chandra ACIS-S (left) and ROSAT HRI (right) observations.
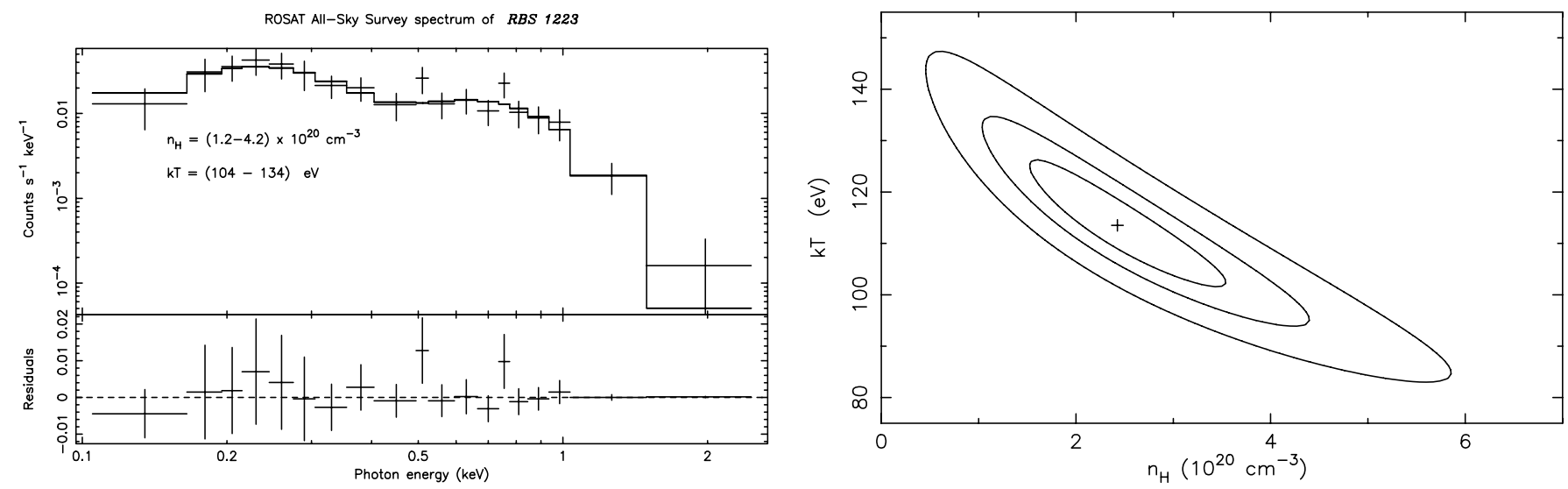

Fig. 4. A blackbody fit and $68 \%, 90 \%$ and $99 \%$ confidence contours $\left(\Delta \chi^{2}=3.5,6.3\right.$, and 11.3 , respectively) to the ROSAT PSPC spectrum of RBS 1223 .

properties of RBS 1223. After extracting (CIAO tool "dmextract") the spectrum of RBS 1223 and the nearby background, we fitted the spectrum with an absorbed blackbody model using the XSPEC V11.0 package.

The ACIS-S instrumental response is not yet known in the energy range $<0.45 \mathrm{keV}$ (see caveats page at http://asc.harvard.edu/cda/caveats.html). Hence, the absorption column density $\left(N_{\mathrm{H}}\right)$ cannot be well constrained by the Chandra observation. The ROSAT PSPC spectra based on All-Sky Survey observation extends down to the $0.1 \mathrm{keV}$. We therefore in our fitting analysis first of all used only ROSAT PSPC data in order to determine $N_{\mathrm{H}}$ and then fixing that value to fit Chandra ACIS-S data in the energy range $0.45-1.5 \mathrm{keV}$, since no photons above $1.5 \mathrm{keV}$ were detected.

The extracted spectrum and corresponding fit of ROSAT All-Sky Survey observation is shown in Fig. 4. The best fit parameters are a column density of $N_{\mathrm{H}}=$ $(2.43 \pm 1.06) \times 10^{20} \mathrm{~cm}^{-2}$, and a temperature $k T_{\mathrm{BB}}=$ $(113.7 \pm 11.8) \mathrm{eV}$ with formal $1 \sigma$ errors.

With the fixed value of $N_{\mathrm{H}}$, the Chandra ACIS-S spectrum of RBS 1223 can be represented by blackbody radiation with temperature $k T_{\mathrm{BB}}=(90.6 \pm 0.8) \mathrm{eV}$ with formal $1 \sigma$ errors (Fig. 5).

The total galactic column density in the direction of RBS 1223 is $N_{\mathrm{H} \text {,gal }}=2.1 \times 10^{20} \mathrm{~cm}^{-2}$, according to the Dickey \& Lockman (1990) maps of HI, averaged into $1^{\circ} \times$ $1^{\circ}$ bins (routine $n h$ in FTOOLS).

In Fig. 6, we show the "on-pulse" spectrum which is defined by averaging over the phase interval $(0.2-0.6)$, i.e. it includes the maximum of the light curve in Fig. 3. For comparison, we also show the "off-pulse" spectrum, obtained by averaging over the remaining phases. On- and off-pulse spectra were rebinned such that each spectral bin contains at least 25 photons. A spectral fit, again with fixed column density $N_{\mathrm{H}}=2.43 \times 10^{20} \mathrm{~cm}^{-2}$, yield bestfit blackbody temperatures of $k T($ on $)=93 \pm 3 \mathrm{eV}$ and $k T$ (off) $=88 \pm 3 \mathrm{eV}$ with formal $3 \sigma$ errors, indicating a trend that the source is hotter at maximum phase (Fig. 6).

\section{Discussion}

The detection of a periodic signal with $P \simeq 5.16$ s proves that RBS 1223is a neutron star. Our determination of 


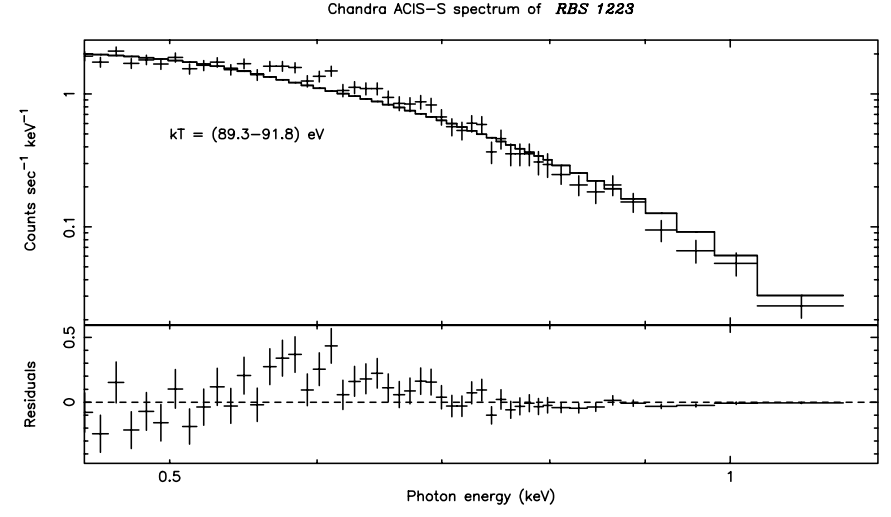

Fig. 5. A blackbody fit to the Chandra ACIS-S spectrum of RBS 1223. A best-fit blackbody temperature is $k T=$ $(91 \pm 1) \mathrm{eV}\left(N_{\mathrm{H}}=(2.43 \pm 1.06) \times 10^{20} \mathrm{~cm}^{-2}\right)$.

$\dot{P} \simeq(0.7-2.0) \times 10^{-11} \mathrm{~s} \mathrm{~s}^{-1}$ shows that RBS 1223 is spinning-down very rapidly. Assuming a spin-down law of the type $\dot{f} \sim-f^{n}(f=1 / P$ is the pulsar rotation frequency, $\dot{f}$ is its derivative, and $n=\ddot{f} f /(\dot{f})^{2}$ is the "braking index") with a constant magnetic moment gives the age of the pulsating neutron star,

$\tau=\frac{P}{(n-1) \dot{P}}\left[1-\left(\frac{P_{0}}{P},\right)^{n-1}\right]$

where $P_{0}$ is a period of the pulsar at birth.

Usually, an oblique rotating vacuum dipole model is assumed, for which $n=3$ (Manchester \& Taylor 1977). If $P_{0}$ is much smaller than $P$, the expression for the age determination reduces to $\tau=P /(2 \dot{P}) \equiv \tau_{\mathrm{c}}$, the characteristic age of a pulsar.

Thus, the characteristic age of RBS 1223 is $\tau_{\mathrm{c}} \simeq$ (6000-12000) yr.

Assuming a neutron star radius of $10^{6} \mathrm{~cm}$ and a moment of inertia of $10^{45} \mathrm{~g} \mathrm{~cm}^{2}$, the dipole magnetic field strength at the pole is

$B_{\text {pole }}=6.4 \times 10^{19} \sqrt{P \dot{P}} \simeq(3.5-6.5) \times 10^{+14} \mathrm{G}$,

well above the so-called "quantum critical field" value $B_{\mathrm{c}} \equiv \frac{m_{\mathrm{e}}^{2} c^{3}}{e \hbar}=4.4 \times 10^{13} \mathrm{G}$. This indicates that RBS 1223 could be a highly magnetized young neutron star.

There has been growing recognition in the literature of a population of highly magnetized neutron stars. The circumstantial evidence for this class comes from studies of soft gamma-ray repeaters (SGRs) and anomalous X-ray pulsars (AXPs).

SGRs are neutron stars showing multiple bursts of gamma-rays with rather soft spectra (e.g. Hurley 2000 for a recent review). The spin periods of SGRs are clustered in the interval 5-8 s. They all appear to be associated with supernova remnants, which limits their average age to approximately $20 \mathrm{kyr}$. The angular offsets of the SGRs from the apparent centers of their associated supernova remnant shells indicate that SGRs are endowed with space velocities $>500 \mathrm{~km} \mathrm{~s}^{-1}$, greater than the space velocities of most radio pulsars (Cordes \& Chernoff 1998).
Chandra ACIS-S on/off pulse spectra of RBS 1223

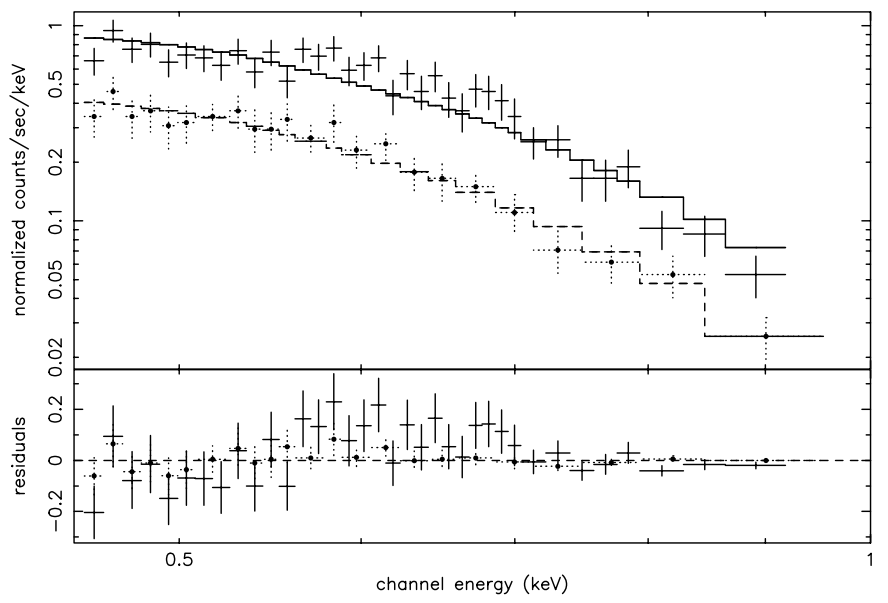

Fig. 6. The on/off pulse spectra of RBS 1223 obtained from Chandra ACIS-S observation. The on-pulse spectrum (upper) corresponds to the phases 0.2 to 0.6 in Fig. 3, while the off-pulse spectrum (lower) is from averaging over the remaining phases. A best-fit blackbody temperatures are $k T$ (on) $=93 \pm 3 \mathrm{eV}$ and $k T($ off $)=88 \pm 3 \mathrm{eV}\left(N_{\mathrm{H}}=2.43 \pm 1.06 \times 10^{20} \mathrm{~cm}^{-2}\right)$.

AXPs are similar to SGRs in that they are radio quiet $\mathrm{X}$-ray pulsars with spin periods clustering in the range $6-12 \mathrm{~s}$, and have similar persistent $\mathrm{X}$-ray luminosities as the SGRs $\left(\sim 10^{35} \mathrm{ergs} \mathrm{s}^{-1}\right.$, see e.g. Stella et al. 1998). Most of the AXPs appear to be associated with supernova remnants, and therefore they are also thought to be young neutron stars like the SGRs. The spin periods of both AXPs and SGRs are increasing with time (spinningdown), and show no evidence for intervals of decreasing spin period (spin-up), although the spin-down rates of many of the SGRs and AXPs appear to be variable or "bumpy" (e.g. Woods et al. 2000).

In addition, it is worth noting that some of the SGRs and AXPs are also located well outside apparently associated supernova remnants (Marsden et al. 2001). Gaensler et al. (2001) argue that they may not be associated at all with these supernova remnants.

Our values of $P$ and $\dot{P}$ are suggestive, that RBS 1223 might belong to either one of the two classes of unusual X-ray pulsars (Fig. 7).

However, it is not excluded also that RBS 1223 may have a conventional $\left(\sim 10^{12} \mathrm{G}\right)$ magnetic field strength and identified as a neutron star in the propeller phase, i.e. powered by inflow of matter from a disk around it (van Paradijs et al. 1995; Chatterjee et al. 2000; Alpar 2001)

Due to the lack of an identification at any other wavelength, the distance of RBS 1223 and hence its luminosity is quite uncertain.

Assuming a size of the X-ray emitting region of 3-10 km, our best fit spectral parameters indicate a distance of $450-1500 \mathrm{pc}(\sim 1.2 \mathrm{kpc}$ assumed by Motch 2000$)$.

Our determination of the absorption column density $N_{\mathrm{H}}=(0.6-6.6) \times 10^{20} \mathrm{~cm}^{-2}(3 \sigma)$ (see Fig. 4) poses no further constraint on the distance of RBS 1223. 


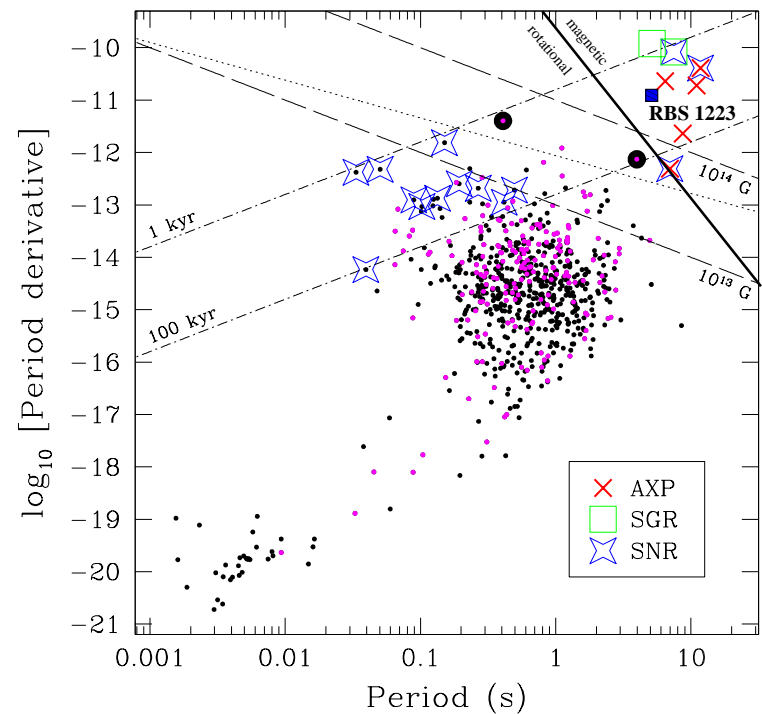

Fig. 7. Plot of $\dot{P}$ versus $P$ for radio pulsars (dots), anomalous X-ray pulsars (AXPs), and soft gamma-ray repeaters (SGRs). The dotted line shown between the lines for $B=10^{13}$ and $10^{14} \mathrm{G}$ indicates a hypothesized approximate theoretical boundary (Baring \& Harding 1998) separating radio-loud and radio-quiet neutron stars due to effects relating to magnetic fields close to the critical field $B_{\mathrm{c}}$ (from Camilo et al. 2000). RBS 1223 is identified by filled box. A solid line is indicating where magnetic field energy and rotational energy losses are equal $\left(\dot{E}_{\mathrm{mag}}=-\frac{1}{6} \dot{B}_{\mathrm{pole}} B_{\mathrm{pole}} R^{3}\right.$ and $\dot{E}_{\mathrm{rot}}=4 \pi^{2} I f \dot{f}$, Heyl \& Kulkarni 1998).

The existence of diffuse high galactic latitude cirruses (at the distance of $100-200$ pc, see Magnani et al. 1996) C9363 and C9383, which extend to the position of RBS 1223 (clearly seen in both IRAS $60 \mu \mathrm{K}$ and IRAS $100 \mu \mathrm{K}$ images, and having column density $N_{\mathrm{H}} \sim$ $(3-4) \times 10^{20} \mathrm{~cm}^{-3}$, Reach et al. 1993) suggests that RBS 1223 might be a nearby $(\sim 100-200$ pc) or rather distant $(\sim 700-1500 \mathrm{pc})$ object.

However, the absence of any obvious supernova remnant in the general direction of this source (Green 2000) and the fact that such a close and young supernova remnant would not remain undetected in the $\mathrm{X}$-rays, suggests the possibility that the source has a very large transverse velocity and it may have migrated far from its original position.

In this context it is worth mentioning that RBS 1223 was not detected as a radio source during the VLA FIRST survey at $1.4 \mathrm{GHz}$ (White et al. 1997) indicating a radioquiet nature. The upper limit of radio flux at the source position is $0.94 \pm 0.138 \mathrm{mJy} /$ beam.

The position of RBS 1223 at the high galactic latitude of $b^{\mathrm{II}}=+83^{\circ}$ imposes an additional restriction on its distance. If RBS 1223 would be at a distance $\sim 1000 \mathrm{pc}$ and would be born in the galactic plane, it must have traveled with a transverse velocity $v_{\mathrm{t}} \sim 1000 \mathrm{~km} \mathrm{~s}^{-1}$ for about $t \sim 10^{6}$ yr. On the other hand, blackbody temperatures in excess of $50 \mathrm{eV}$ imply ages younger than $\sim 10^{6}$ yr for standard cooling curves (see Tsuruta 1998). This suggests a much smaller distance than $1 \mathrm{kpc}$.
Assuming a distance of the source of $\sim 1000 \mathrm{pc}$ and $k T_{\mathrm{BB}}=91 \mathrm{eV}$, we determine the unabsorbed luminosity to be

$$
L_{\mathrm{X}} \sim 4.1 \times 10^{32} \operatorname{ergs~s}^{-1} \text {. }
$$

\section{Conclusions}

We analyzed Chandra ACIS-S and an archival ROSAT HRI observations of the isolated neutron star candidate RBS 1223 and found a period $P \sim 5.16 \mathrm{~s}$. We also found that the neutron star spins down at $\dot{P} \sim(0.7-2.0) \times$ $10^{-11} \mathrm{~s} \mathrm{~s}^{-1}$ with some remaining uncertainty on the spin down rate. Confirmation of our result requires further X-ray observations of RBS 1223 .

The nature of RBS 1223 remains uncertain, even if the spin down of the pulsar can be confirmed independently. It might be a young neutron star either with a very strong magnetic field (magnetar) or a neutron star with a more conventional magnetic field of $\sim 10^{12} \mathrm{G}$ in the propeller phase powered by inflow of matter from a surrounding disk.

The absence of a nearby supernova remnant and the "characteristic age" of a pulsar suggest a high space velocity to bring RBS 1223 away from its progenitor supernova remnant.

Additional constraints on its nature can be derived from detailed $\mathrm{X}$-ray spectroscopy using upcoming XMM data and/or an $\mathrm{X}$-ray proper motion study which we have already initiated.

Acknowledgements. This project was supported by the Bundesministerium für Bildung und Forschung through the Deutsches Zentrum für Luft- und Raumfahrt e.V. (DLR) under grant number 50 OR 97068 .

\section{References}

Alpar, M. A. 2001, ApJ, 554, 1245

Baring, M. G., \& Harding, A. K. 1998, ApJ, 507, L55

Blaes, O., \& Madau, P. 1993, ApJ, 403, 690

Brazier, K. T. S. 1994, MNRAS, 268, 709

Bretthorst, L. 1988, Bayesian Spectrum Analysis and Parameter Estimation (Springer-Verlag), 1

Buccheri, R., Bennett, K., Bignami, G. F., et al. 1983, A\&A, 128,245

Camilo, F., Kaspi, V. M., Lyne, A. G., et al. 2000, ApJ, 541, 367

Chatterjee, P., Hernquist, L., \& Narayan, R. 2000, ApJ, 534, 373

Cordes, J. M., \& Chernoff, D. F. 1998, ApJ, 505, 315

Dickey, J. M., \& Lockman, F. J. 1990, Ann. Rev. Astron. Astrophys., 28, 215

Gaensler, B. M., Slane, P. O., Gotthelf, E. V., \& Vasisht, G. 2001 [astro-ph/0104228]

Green, D. A. 2000, A Catalogue of Galactic Supernova Remnants (2000 August version), Mullard Radio Astronomy Observatory (Cambridge, United Kingdom), available at http://www.mrao.cam.ac.uk/surveys/snrs/

Gregory, P., \& Loredo, T. 1992, ApJ, 398, 146 
Gregory, P., \& Loredo, T. 1993, in Proc. 12th Int. Conf. on Maximum Entropy and Bayesian Methods, ed. A. Mohammad-Djafari, \& G. Demoment (Dordrecht: Kluwer), 225

Gregory, P., \& Loredo, T. 1996, ApJ, 473, 1059

Haberl, F., Motch, C., Buckley, D. A. H., Zickgraf, F.-J., \& Pietsch, W. 1997, A\&A, 326, 662

Haberl, F., Motch, C., \& Pietsch, W. 1998, Astron. Nachr., 319, 97

Haberl, F., Pietsch, W., \& Motch, C. 1999, A\&A, 351, L53

Heyl, J. S., \& Kulkarni, S. R. 1998, ApJ, 506, L61

Horne, J. H., \& Baliunas, S. L. 1986, ApJ, 302, 757

Hurley, K. 2000 in Proceedings of the 5th Huntsville GRB Symposium (AIP Press: New York), in press [astro-ph/9912061]

Jaynes, E. T. 1987, Bayesian Spectrum and Chirp Analysis, in Maximum Entropy and Bayesian Spectral Analysis and Estimation Problems, ed. C. Ray Smith, \& G. J. Erickson, (D.Reidel, Dordrech - Holland), 1

Kovacs, G. 1981, Ap. Sp. Sci, 78, 175

Magnani, L., Hartmann, D., \& Speck, B. G. 1996, ApJSS, 106, 447

Manchester, R. N., \& Taylor, J. H. 1977, Pulsars (San Francisco: Freeman)

Marsden, D., Lingenfelter, R. E., Rothschild, R. E., \& Higdon, J. C. 2001, ApJ, 550, 397

Motch, C., Haberl, F., Zickgraf, F.-J., Hasinger, G., \& Schwope, A. D. 1999, A\&A, 351, 177

Motch, C., \& Haberl, F. 1998, A\&A, 333, L59

Motch, C. 2000 [astro-ph/0008485]
Narayan, R., \& Ostriker, J. P. 1990, ApJ, 352, 222

Neuhäuser, R., \& Trümper, J. 1999, A\&A, 343, 151

Reach, W. T., Helles, C., \& Koo, B. 1993, ApJ, 412, 127

Scargle, J. 1982, ApJ, 263, 835

Schwope, A. D., Hasinger, G., Schwarz, R., Haberl, F., \& Schmidt, M. 1999, A\&A, 341, L51

Schwope, A. D., Hasinger, G., Lehmann, I., et al. 2000, Astron. Nachr., 321, 1

Stella, L., Israel, G. L., \& Mereghetti, S. 1998, Ad. Sp. R., 22, 1025

Treves, A., \& Colpi, M. 1991, PASP, 112, 297

Treves, A., Turola, R., Zane, S., \& Colpi, M. 2000, PASP, 112, 297

Tsuruta, S. 1998, Physics Rep., 292, 1

van Paradijs, J., Taam, R. E., \& van den Heuvel, E. P. J. 1995, A\&A, 299, L4

Voges, W., Aschenbach, B., Boller, Th., et al. 1999, A\&A, 349, 389

Walter, F. M., Wolk, S. J., \& Neuhäuser, R. 1996, Nature, 379 233

Walter, F. M. 20001, ApJ, 549, 433

White, R. L., Becker, R. H., Helfand, D. J., \& Gregg, M. D. 1997, ApJ, 475, 479, A source catalogue of Faint Images of the Radio Sky at Twenty-cm (2000 July version), available at http://sundog.stsci.edu

Woods, P. M., Kouveliotou, C., Finger, M. H., et al. 2000, ApJL, 535, L55

Zimmermann, H. U., Boese, G., Becker, W., et al. 1998, EXSAS Users Guide, MPE Report, ROSAT Scientific Data Center (Garching) 